\title{
USO DEL DIMINUTIVO EN ECUADOR: SIGNO DE CONFIANZA EN LA RELACIÓN MÉDICO-PACIENTE, ENFERMERO- PACIENTE
}

\section{USE OF DIMINUTIVES IN ECUADOR: SIGN OF TRUST IN THE DOCTOR-PATIENT, NURSE-PATIENT RELATIONSHIP}

\author{
Estupiñan-Lince María Lorena ${ }^{1 *}$ \\ ${ }^{1}$ Universidad Rusa de la Amistad de los Pueblos, Facultad de Filología. Moscú, Rusia.
}

*Correo: 1032188152@pfur.ru

\begin{abstract}
Resumen
En Ecuador, utilizar diminutivos en el lenguaje formal y familiar es muy popular y por lo tanto también en ámbitos como el área de la salud. Este estudio tiene como objetivo identificar los principales diminutivos utilizados por el personal de la salud con los pacientes adultos y las razones por las cuales se emplean. Además, conocer la opinión de los pacientes respecto al uso de los diminutivos en las consultas médicas. Por medio de una encuesta en línea se obtiene la información para comprobar la hipótesis. Los resultados permiten conocer que el diminutivo más utilizado según el personal de la salud ecuatoriano es "un ratito" y el diminutivo más escuchado por los pacientes es parte de la frase "deme su bracito". Las principales razones por las cuales el personal de la salud utiliza los diminutivos en las consultas son: 1. Para dar confianza a sus pacientes, 2. por cortesía. Al respecto, la opinión de los pacientes está dividida: primero están aquellos que efectivamente cuando escuchan diminutivos se sienten en un ambiente de confianza, luego, los que manifiestan que les da igual escuchar diminutivos, y por último los que prefieren que no se utilicen diminutivos con ellos.
\end{abstract}

Palabras clave: diminutivos, personal de la salud, Ecuador, signo, confianza, pacientes, relación.

\begin{abstract}
Using diminutives in formal and informal language in Ecuador is very popular and therefore also in areas such as the health area. This paper aims to identify the main diminutives used by medical staff with adult patients and their main reasons to use them. Also, to know patient opinion about the use of diminutives at medical appointments. Through an online survey, information is obtained to verify the proposed hypothesis. Results allow to know: the most used diminutive according to the medical Staff is "un ratito" and the diminutive most listened by patients is part of the phrase "deme su bracito". The main reasons why medical staff use diminutives at medical appointments are: 1 . Give trust to their patients, 2. as a polite set expression. Relate to this topic patient opinion is divided: chiefly, there are those who actually when they listen diminutives feel in a confidencebuilding environment, others who say that they do not care about listening diminutives and finally, those who prefer that medical staff does not use diminutives with them.
\end{abstract}

Keywords: diminutives, medical staff, Ecuador, sign, trust, patients, relationship.

Información del manuscrito:

Fecha de recepción: 08 de mayo de 2020.

Fecha de aceptación: 09 de julio de 2020.

Fecha de publicación: 10 de julio de 2020. 


\section{Introducción}

En el diccionario de la Real Academia Española se define al diminutivo como: "dicho de un sufijo, que expresa disminución, atenuación 0 intensidad de lo denotado por el vocablo al que se une, o que valora afectivamente su significación" (RAE, 2019).

Los ecuatorianos están muy inclinados al uso de los diminutivos (Córdova, 1995), al hablar no solo con los niños, sino también con los adultos.

En Ecuador, los diminutivos se forman con los sustantivos: palomita, los adjetivos: bonita, con los adverbios para expresar el hecho de que la acción se llevará a cabo en pocos minutos: aquisito (Espinoza, 2000), también se aplican a los números cardinales: dosito (García, Meilán, Martínez, 2004) y hasta con los verbos: corriendito.

El tema del uso de los diminutivos en el Ecuador actualmente es objeto de discusión. Por un lado están aquellos que argumentan que su uso es exagerado e incluso les incomoda escucharlos en todas partes, incluidos los hospitales y centros de salud (Mera, 2019) y por otro lado están los que consideran que esta forma de hablar en Ecuador se convierte en una característica de su español, debido a la fuerte influencia del quichua (Catta, 1994) y (Espinoza, 2000) y a factores temperamentales de sus habitantes (Cordero de Espinoza, 2019).

El uso de los diminutivos en el habla de los ecuatorianos es muy popular, extendiéndose hasta el ambiente del área de la salud, sobre todo cuando a través de los años el personal sanitario ha tratado de mejorar la relación con sus pacientes.

Un suceso que marcó el final del siglo XX y el inicio del siglo XXI fue la nueva concepción de lo que la relación ideal médico-paciente debe ser, resumiéndose en seis ejes: "1. Relación de compromiso: confianza, seguridad. 2. Relación de amistad: confidencialidad, intimidad, secreto. 3. Relación ético-narrativa: tiempo, esperanza, oportunidad, etc. 4. Relación técnica: buen diagnóstico, pronóstico, tratamiento. 5. Relación deliberativa: prudencia. 6. Relación contractual: derechos y deberes" (Chivato, 2019).

Los diminutivos pueden exprezar una extendida gama de emociones: 
amor, cariño, simpatía, cercanía, pequeñez, ternura, compasión, pero también desprecio 0 ironía. La influencia del idioma quichua en el español de Ecuador se evidencia principalmente a la hora de manisfestar cortesía, simpatía o ternura por medio de lo que en quichua se denominan los "Diminutivos cariñosos": -itu/-ita, zitu/ -situ, -shitu -hua/-huazu, huahua, -cu, -gu, -nai/nallai, -lla. Por ejemplo: jatunllazitu: grandecito (Catta, 1994).

Al respecto, al diminutivo cariñoso utilizado en el Ecuador, también se le denomina "El diminutivo que no disminuye" debido a la gran carga de afectividad que conlleva su uso, lejos de la intención de disminuir el tamaño del objeto (Córdova, 1995).

Sin embargo, el uso de los diminutivos es un tema de discusión en la actualidad. Por este motivo este estudio tiene tres objetivos principales: 1. Investigar los principales diminutivos utilizados por el personal de la salud en el Ecuador. 2. Identificar las razones por las cuales el personal de la salud en el Ecuador utiliza diminutivos para hablar con sus pacientes. 3. Conocer la opinión de los pacientes ecuatorianos al escuchar que el personal de la salud utiliza diminutivos en las consultas.

La hipótesis de este estudio es: si el personal de la salud de Ecuador utiliza diminutivos en las consultas, los pacientes se sentirán en confianza.

\section{Materiales y métodos}

El tipo de investigación fue cualitativa por la información personal sobre cada uno de los encuestados que se recolectó en las preguntas abiertas de la encuesta. Pero también fue cuantitativa, por el tipo de preguntas cerradas que se formularon y cuyas respuestas pudieron ser cuantificables, tabularse, analizarse y dar porcentajes importantes para el estudio.

El nivel de esta investigación fue explorativo. Esta investigación se centró en analizar un aspecto real del español hablado en Ecuador, en este caso limitado al personal sanitario, esperando que posteriores investigaciones también puedan analizar este tema de estudio con mayor profundidad. 
Las encuestas se enviaron por WhatsApp a un total de 150 ecuatorianos, de los cuales 107 aceptaron participar en el estudio. 32 profesionales respondieron a la primera encuesta que era solo para personal de la salud, esto constituyó la muestra 1 de este estudio (22 doctores, 4 odontólogos, 3 enfermeros, 1 tecnólogo radiólogo, 1 terapista del lenguaje y 1 laboratorista) Esta muestra tuvo 22 participantes provenientes de la región Costa, 9 de la región Sierra y 1 participante del Oriente. No hubo nadie de la región Insular. En esta muestra 22 participantes eran mujeres, 8 hombres y 2 participantes prefirieron no escribir el género. El $59,37 \%$ de los participantes tenían edades comprendidas entre los 30 a los 40 años de edad, y el 40,62\% entre los 25 a los 29 años de edad.

75 participantes respondieron a la segunda encuesta, este grupo constituyó la muestra 2 de este estudio (19 profesores, 18 ingenieros, 4 psicólogos, 3 economistas, 2 biólogos, 2 tecnólogos, 1 secretario, 1 geólogo, 1 comercializador, 1 guía, 1 diseñador, 1 empleado, 1 asesor legal, 13 estudiantes universitarios, 4 personas sin estudios superiores y 3 participantes no indicaron su nivel de educación en la hoja de respuestas). Esta muestra tuvo 44 participantes provenientes de la región Costa, 30 de la región Sierra y 1 de la región Insular. No participó nadie del Oriente. En esta muestra 43 participantes eran mujeres y 32 hombres. El $41,33 \%$ de los participantes tenían edades comprendidas entre los 31 a los 40 años de edad, el $40 \%$ entre los 18 a los 30 años de edad, el $12 \%$ de los participantes tenían más de 50 años de edad, el 6,66\% entre los 41 a los 50 años de edad.

La variable independiente de esta investigación es el uso de los diminutivos por el personal de la salud en el Ecuador.

La variable dependiente es la confianza que sienten los pacientes ecuatorianos al escuchar diminutivos en el lenguaje oral utilizado en una consulta.

El instrumento utilizado fue una encuesta realizada en Google Forms, la cual se envió por WhatsApp. Las encuestas se realizaron bajo las normas de confidencialidad de los datos de los participantes. 
Para el análisis de los datos de las encuestas se realizaron los siguientes pasos: 1. Se Verificó la calidad de los datos. 2. Se tabularon los datos. 3. Se analizaron los números. 4. Se compararon los resultados. 5. Se obtuvieron las conclusiones.

\section{Resultados}

\subsection{Resultados de la encuesta a} la muestra 1 (Personal de la salud)

\section{El diminutivo más utilizado por el personal de la salud en el Ecuador}

El $34,37 \%$ de los participantes utiliza el diminutivo "un ratito", el $12,5 \%$ de los participantes no emplea diminutivos con sus pacientes, el 9,37\% "abra la boquita", y otro 9,37\% "deme su bracito", el 6,25\% "abra los ojitos", un 6,25\% "no mueva los piecitos", un 6,25\% "quédese quietita", el 6,25\% "un minutito", y el $6,25 \%$ no respondió nada.

Razones por las que el personal de la salud ecuatoriano utiliza diminutivos con sus pacientes

El $65,62 \%$ de los participantes utiliza diminutivos para dar confianza a sus pacientes, el $25 \%$ de los participantes prefiere no utilizar diminutivos con sus pacientes, el $3,12 \%$ los emplea por cortesía, otro $3,12 \%$ lo hace de manera involuntaria, y otro $3,12 \%$ no respondió nada.

¿Se pueden sentir ofendidos los pacientes al escuchar diminutivos en una consulta?

El $56,25 \%$ cree que sus pacientes no se sienten ofendidos al escuchar diminutivos, el $31,25 \%$ cree que tal vez sí, el $12,5 \%$ cree que sí se sienten ofendidos los pacientes.

\subsection{Resultados de la encuesta a la muestra 2}

El diminutivo más escuchado por los pacientes en el Ecuador

El 28\% de los participantes escogió "deme su bracito", el 22,6\% de los participantes respondió que nunca han utilizado diminutivos con ellos en instituciones de salud, el 10,66\% "alce los bracitos", el $8 \%$ utiliza "abra los ojitos", el 6,66\% "quédese quietito", el $4 \%$ "deje su ropita", otro $4 \%$ "le voy a meter esta agujita", otro $4 \%$ "mueva la cabecita", el 2,66\% "abra la boquita", otro 2,66\% "cúbrase con la batica", otro 2,66\% "no mueva los piecitos", otro $2,66 \%$ 
no respondió nada, y el 1,33\% "más tarde le traemos un juguito".

\section{¿Qué han sentido los pacientes al escuchar diminutivos en una consulta?}

El $42,66 \%$ de los participantes se ha sentido en un clima de confianza, al $33,33 \%$ le da igual que usen diminutivos o no, el $20 \%$ prefiere que le hablen sin diminutivos, el $2,66 \%$ escribió que ha escuchado usar diminutivos con niños o con adultos mayores y el 1,33\% no respondió nada.

\section{Discusión}

De acuerdo con los resultados obtenidos en este estudio se confirma que el personal de la salud en Ecuador utiliza los diminutivos como recurso inspirador de confianza con los pacientes adultos en primer lugar, segundo por cortesía y tercero de manera involuntaria, aunque hay un $25 \%$ que no utiliza diminutivos con los pacientes.

De acuerdo con los datos obtenidos de la encuesta al personal sanitario, los diminutivos más utilizados son: un ratito, abra la boquita, deme su bracito, abra los ojitos, no mueva los piecitos, quédese quietita y un minutito. Este hallazgo concuerda con el estudio realizado en Ecuador por los estudiantes de la universidad de Cuenca "Expresiones más utilizadas por los jóvenes cuencanos" en el año 2014, en el cual se encontró que el diminutivo más utilizado por los cuencanos es "un ratito". Sin embargo, los pacientes opinaron que los diminutivos que más escuchan en las consultas son: deme su bracito, alce los bracitos, abra los ojitos, quédese quietito, deje su ropita, le voy a meter esta agujita, mueva la cabecita, abra la boquita, cúbrase con la batita, no mueva los piecitos y más tarde le traemos un juguito. Al respecto, se observa que los diminutivos son utilizados principalmente junto con los imperativos, como una forma de suavizar las órdenes 0 las instrucciones que se están diciendo. Esto conlleva un matiz de cortesía al hablar. Tal y como lo explica con un ejemplo la directora de la Academia Ecuatoriana de la Lengua «Si pedimos a la empleada doméstica: María, tráigame un vaso de agua, ella intuirá: -Algo le pasa a la señora, está bravísima. Pero si decimos - 
María, (o Maríta), deme trayendo un vaso de agua, no sea malita? atenderá sin reserva al llamado de sintaxis y significado trastornados, y 'enternecidos' con el diminutivo» (Cordero de Espinoza, 2019).

Se identifica, que el sufijo diminutivo más popular en Ecuador en el área de la salud es -it/o,a. Lo cual coincide con el diminutivo más utilizado en el castellano de España y uno de los diminutivos más utilizados en el quichua de Ecuador, por lo tanto, este es uno de los factores que intervienen para que el uso de los diminutivos en el Ecuador sea tan popular.

La principal razón por la que el personal de la salud utiliza diminutivos es: como recurso para inspirar confianza en los pacientes, sin embargo, solo el $42,66 \%$ de los pacientes confirmaron que se sienten en confianza al escuchar diminutivos.

Los resultados de este estudio pudieran ser diferentes si en investigaciones futuras la muestra 1 constara de un mayor número de participantes provenientes de Galápagos y de la Amazonía. Además, también los resultados pueden variar si el número de participantes de género masculino y género femenino fuera igual 0 aproximado.

\section{Conclusiones}

Es de vital importancia seguir investigando sobre el tema del uso de los diminutivos en Ecuador y sobre todo llegar hasta la publicación de los resultados obtenidos para así contribuir al estudio de la variante del español que se habla en Ecuador por los lingüístas mundialmente.

Es difícil realizar investigaciones sobre el habla de los ecuatorianos cuando Ecuador no cuenta con un Corpus digital en donde los lingüistas o filólogos puedan realizar sus estudios. Ecuador solo cuenta como recurso con los tomos del Diccionario de Ecuatorianismos llamado "El habla de los ecuatorianos" en él se puede realizar investigación, pero al no estar en un corpus digital hay muchas desventajas y limitaciones para los investigadores.

En conclusión, el uso de los diminutivos en el Ecuador, lejos de ser una manera infantilista de hablar, o constituir una muestra de falta de 
educación, es una característica distintiva del habla de los ecuatorianos, tal y como lo afirma la directora de la Academia Ecuatoriana de la Lengua, Susana Cordero de Espinoza. Esta costumbre indiscutiblemente también se ha extendido en las instituciones de la salud y es un signo de confianza en la relación médico-paciente y enfermeropaciente en Ecuador.

Se recomienda, sin embargo, al personal sanitario, emplear los diminutivos con mucha atención, porque no todos los pacientes pueden sentirse a gusto con este trato, aunque la intención sea buena.

\section{Bibliografía}

Andrade, P. et al. (2014). Expresiones más utilizadas por los jóvenes cuencanos. Tostarte.

https://sites.google.com/site/t ostarte1/cultura/expresionesmas-utilizadas-por-losjovenes-cuencanos

Catta, J. (1994). Gramática del quichua Ecuatoriano. Ediciones Abya-Yala.

Cordero de Espinoza, S. (2019). Excelentísimos Lenguarazes. Agencia EFE. https://www.efe.com/efe/espa na/firmas-de-

efe/excelentisimoslenguarazes/500010503932170

Córdova, C. (1995). El habla del Ecuador: Diccionario de Ecuatorianismos. Universidad del Azuay.

Chivato, T. (2019). La relación médico-paciente. Claves para un encuentro humanizado. Midac, SL.

Espinoza, M. (2000). Los mestizos ecuatorianos y las señas de identidad cultural. Trama social editorial.

García S., Meilán A., Martínez H. (2004). Construir bien en español la forma de las palabras. Ediuno.

Mera, A. (2019). ¿Qué es un paciente? EL COMERCIO. https://www.elcomercio.com/o pinion/paciente-opinion-auralucia-mera.html

Real Academia Española (2019). Diminutivo Rae.es. https://dle.rae.es/diminutivo? $m=$ form 